\title{
Low-frequency ultrasound enhances chemotherapy sensitivity and induces autophagy in PTX-resistant PC-3 cells via the endoplasmic reticulum stress-mediated $\mathrm{PI} / 3 \mathrm{~K} / \mathrm{Akt} / \mathrm{mTOR}$ signaling pathway
}

This article was published in the following Dove Press journal: OncoTargets and Therapy

Yuqi Wu'

Xiaobing Liu $^{2}$

Zizhen Qin'

$\mathrm{Li} \mathrm{Hu}$

Xiangwei Wang'

'Department of Urology, Carson International Cancer Center, Shenzhen University General Hospital \& Shenzhen University Clinical Medical Academy Center, Shenzhen University, Shenzhen, People's Republic of China; ${ }^{2}$ Department of Urology, Second Affiliated Hospital, Third Military Medical University, Chongqing, People's Republic of China

Correspondence: Xiangwei Wang Shenzhen University General Hospital, No 1098, Xueyuan Road, Nanshan District, Shenzhen 518000, People's Republic of China

Tel +86 I $7796349 \mid 57$

Fax +86 075521839000

Email winn0324@szu.edu.cn
Background: Sonodynamic therapy (SDT) is an emerging tumor-inhibiting method that has gained attention in cancer therapy in the last several years. Although autophagy has been observed in SDT-treated cancer cells, its role and mechanism of action remain unclear. This study aimed to investigate the effects of low-frequency ultrasound on autophagy and drug-resistance of paclitaxel (PTX)-resistant PC-3 cells via the endoplasmic reticulum stress (ERs)-mediated $\mathrm{PI} 3 \mathrm{~K} / \mathrm{AT} / \mathrm{mTOR}$ signaling pathway.

Methods: CCK- 8 assay was conducted to select the appropriate exposure time for PTXresistant PC-3 cells under low-frequency ultrasound. PTX-resistant PC-3 cells were divided into a control group, PTX group, ultrasound group, ultrasound + PTX group, ultrasound + PTX + autophagy-related gene 5 (Atg5) siRNA group, and ultrasound + 4-PBA (an ERs inhibitor) group. Autophagy was observed by transmission electron microscopy (TEM) and fluorescence microscopy. Cell proliferation was evaluated using CCK-8 assay; apoptosis was detected by flow cytometry. Expression of multiple drug-resistance genes was detected by qRT-PCR. Western blotting was used to detect the expression of ERS-related proteins, autophagy-related proteins, apoptosis-related proteins, and $\mathrm{PI} 3 \mathrm{~K} / \mathrm{AKT} / \mathrm{mTOR}$ pathway-related proteins.

Results: Ten-second exposure was selected as optimal for all experiments. Compared to the PTX group, the level of autophagy, inhibition rate, apoptosis rate, and expression of ERS-related proteins (GRP78) increased, whereas the expression of multiple drug-resistance genes (MRP3, MRP7, and P-glycoprotein), PI3K/AKT/mTOR pathway-related proteins (PI3K, p-AKT, mTORC1), and apoptosis-related proteins $(\mathrm{Bcl}-2, \mathrm{NF}-\kappa \mathrm{B})$ decreased in PTX-resistant PC-3 cells after lowfrequency ultrasound and PTX treatment for $24 \mathrm{~h}$. These trends were more obvious after treatment with Atg5 siRNA, excluding the autophagy level. Post 4-PBA-treatment, the expression of GRP78 and LC3II proteins decreased, whereas that of PI3K, p-AKT, and mTORC1 increased.

Conclusion: Results indicated that ultrasound induces autophagy by ERs-mediated PI3K/AKT/ mTOR signaling pathway in PTX-resistant PC-3 cells; this autophagy acts as a cytoprotector during low-frequency ultrasound-mediated reversal of drug resistance.

Keywords: prostate cancer, multidrug resistance, sonodynamic therapy, autophagy, apoptosis, endoplasmic reticulum stress

\section{Introduction}

Prostate cancer is the most common cancer affecting middle-aged and elderly men and has become the second leading cause of cancer-related deaths in men. ${ }^{1}$ Early-stage 
prostate cancer is primarily treated with radical surgery, cryotherapy, and radiation therapy. Advanced prostate cancer patients are commonly treated with paclitaxel (PTX)-based chemotherapy after failure of androgen deprivation therapy. However, drug resistance can develop when the treatment fails to inhibit prostate cancer progression. Therefore, there is an urgent need to develop new treatment strategies for prostate cancer. $^{2}$

Sonodynamic therapy (SDT) combined with lowfrequency ultrasound has a strong penetrating ability in biological tissues. The application of focused ultrasound is it can focus the sound energy on deep tissues without causing injury. Furthermore, SDT with low-frequency ultrasound contributes to the activation of several ultrasonic-sensitive drugs, such as hematoporphyrin, to achieve non-invasive eradication of solid tumors. ${ }^{3}$ Recent studies reported that the combination of low-frequency ultrasound with chemotherapeutic drugs can enhance chemotherapy sensitivity and reverse drug resistance in tumor cells. ${ }^{4}$

Autophagy has been observed in tumor cells during application of low-frequency ultrasound to irradiate nasopharyngeal carcinoma cells and prostate cancer cells. ${ }^{5,6}$ Nevertheless, the role of autophagy and its associated mechanisms of action remain unclear. Autophagy is an evolutionarily conserved process. Autophagosomes perform the recovery of amino acids and energy by encapsulating cytoplasm and organelles and degrading them in the lysosomes. The role of autophagosomes in the survival and death of cancer cells has always been controversial. Extensive studies have demonstrated that autophagy acts as a protective mechanism against cancer. Autophagy can protect cancer cells from various stimuli, such as amino acid deficiency, hypoxia, DNA and mitochondrial damage, and oxidative stress. ${ }^{7}$ However, autophagy has also been reported to inhibit the proliferation of tumor cells and induce cell death (type II programmed cell death) by acting in cooperation with apoptosis. ${ }^{8}$ Therefore, examining the role of autophagy in low-frequency ultrasound-assisted chemotherapy is necessary to elucidate the mechanisms by which drug resistance can be reversed using low-frequency ultrasound. This way, new targets can be identified and novel strategies for reversing drug resistance in prostate cancer can be developed.

\section{Materials and methods}

\section{Cell culture and ultrasound treatment}

The PTX-resistant PC-3 cell line was purchased from the Guangxi Nanning Longevity Biological Technology Co., Ltd. (Guangxi, China). The use of PTX-resistant PC-3 cell line has been approved by Second Affiliated Hospital of
Third Military Medical University. Instruments for ultrasound treatment (Metron, AA170 type) were provided by the Third Military Medical University. Cells were incubated in RPMI-1640 medium (Thermo Fisher Scientific) supplemented with 10\% fetal bovine serum (Thermo Fisher Scientific) and subsequently cultured in a $5 \% \mathrm{CO}_{2}$ incubator with saturated humidity at $37^{\circ} \mathrm{C}$. A low-frequency ultrasound probe using degassed sterile water as a coupling agent was used to irradiate the bottom of a six-well plate containing $2 \mathrm{~mL}$ of the cell suspension $\left(5 \times 10^{5}\right.$ cells $\left./ \mathrm{mL}\right)$. In the present study, PTX-resistant PC-3 cells were exposed to continuous ultrasound with a frequency of $1 \mathrm{MHz}$, and the spatial average intensity was set to $1.2 \mathrm{~W} / \mathrm{cm}^{2}$.

\section{Treatment groups}

PTX-resistant PC-3 cells in the logarithmic phase were divided into the control group (without treatment), PTX group (treated with 91.44 nM PTX; Solarbio), ultrasound group (treated with low-frequency ultrasound), and ultrasound + PTX group (treated with low-frequency ultrasound and $91.44 \mathrm{nM}$ PTX). The ultrasound + PTX + Atg5 siRNA group was treated with low-frequency ultrasound and 91.44 nM PTX after transfection with Atg5 siRNA lentivirus (GenePharma). The ultrasound + 4-PBA group was treated with low-frequency ultrasound and $10 \mathrm{mM}$ 4-phenylbutyric acid (4-PBA) (Sigma-Aldrich Co.). The ultrasound + dimethyl sulfoxide (DMSO) group was treated with low-frequency ultrasound and $10 \mathrm{mM}$ DMSO (Sigma-Aldrich Co.).

\section{Ultrasonic cytotoxicity}

PTX-resistant PC-3 cells were treated with low-frequency ultrasound for varying time periods $(0,10,20,30,40,50$, and 60 seconds). After the treated cells $\left(5 \times 10^{5}\right.$ cells/well) were incubated in a 96 -well plate at $37^{\circ} \mathrm{C}$ for 24 hours, the cell culture medium was removed. Each well was added with Cell-Counting Kit 8 (CCK-8, $10 \mu \mathrm{L}$; Dojindo) solution dissolved in $100 \mu \mathrm{L}$ of medium and incubated for 1 hour in the dark. Then, the OD of each well at a $450 \mathrm{~nm}$ was measured using a microplate reader. The percentage of cytotoxicity was calculated based on the following equation: Cytotoxicity $(\%)=(\mathrm{OD}$ control group $-\mathrm{OD}$ ultrasound group $) / \mathrm{OD}$ control group $\times 100 \%$.

\section{Transmission electron microscopy}

Fixed cells were post-fixed in $2 \% \mathrm{OsO}_{4}$, dehydrated in graded alcohol, and flat embedded in Epon 812 (Electron Microscopy Sciences). Ultrathin sections $(80 \mathrm{~nm})$ were prepared, stained with uranyl acetate and lead citrate, and examined using an electron microscope (Hitachi). 
Table I Primers used for qRT-PCR analysis

\begin{tabular}{|c|c|c|}
\hline Gene & Forward primer & Reverse primer \\
\hline$\beta$-Actin & 5'-AGCGAGCATCCCCCAAAGTT-3' & 5'-GGGCACGAAGGCTCATCATT-3' \\
\hline Bcl-2 & 5'-GCCTTCTTTGAGTTCGGTGG-3' & 5'-GAAATCAAACAGAGGCCGCA-3' \\
\hline MRP3 & 5'-TTCCTGGTGGCTGGGCTGATGT-3' & 5'-GACGCACGTTTGACTGAGTTGG-3' \\
\hline MRP7 & 5'-AGAGTACACCTGTGACCTGC-3' & $5^{\prime}$-GAAGAGCACCAACAACAGGG-3' \\
\hline P-glycoprotein & 5'-GAGCCTACTTGGTGGCACAT-3' & 5'-TCCTTCCAATGTGTTCGGCA-3' \\
\hline
\end{tabular}

Abbreviation: qRT-PCR, quantitative reverse-transcription PCR.

\section{Western blotting}

The anti-PI3 Kinase p110 beta antibodies were used at a 1:600 dilution. The anti-GRP78 antibodies, anti-AKT1 (phospho S473) antibodies, anti-mTOR (phospho S2481) antibodies, anti-LC3B antibodies, anti-APG5L/ATG5 antibodies, anti-NF-kB p65 antibodies, and anti-Bcl-2 antibodies were used at 1:1,000 dilution. The anti-beta actin antibodies were used at 1:200 dilution. All the antibodies were obtained from Abcam (Cambridge, UK).

\section{Apoptosis assay}

Apoptosis was detected using an Annexin V-PE Apoptosis Detection Kit (BD Pharmingen). Cells in the logarithmic growth phase were harvested and washed twice in PBS. Then, $1 \times 10^{6}$ cells were counted and washed twice in PBS before resuspension in $1 \times$ binding buffer. Cells were incubated with PE Annexin V $(5 \mu \mathrm{L})$ and 7-AAD $(5 \mu \mathrm{L})$ on ice for 30 minutes to allow cell staining, followed by the addition of $400 \mu \mathrm{L}$ of $1 \times$ binding buffer to each sample.

\section{Cell proliferation assay}

Cell proliferation was evaluated using a CCK- 8 assay according to the manufacturer's instructions (Donjindo). PTX-resistant PC-3 cells in each well were subjected to varying treatments for 24 hours, seeded in 96-well plates (2,000 cells/well), and added with $10 \mu \mathrm{L}$ of CCK-8 solution at the indicated time points after transfection. Cells were then incubated for an additional 2 hours. The absorbance values of the cells were then measured at $450 \mathrm{~nm}$ on a Multiskan FC Microplate Photometer (Thermo Fisher Scientific).

\section{qRT-PCR}

Total RNA was extracted from harvested cells with TRIzol (Thermo Fisher Scientific). Briefly, for the detection of Bcl-2, MRP3, MRP7, and P-glycoprotein, $1 \mu \mathrm{g}$ of total RNA per sample was converted to cDNA using a cDNA Synthesis Kit (Thermo Fisher Scientific). The cDNAs were amplified and detected using a SYBR Green PCR kit (Qiagen). GAPDH was used as an endogenous control. For detection of the mRNA, cDNA products were synthesized using the miScript
Reverse Transcription Kit (Qiagen). Sequence-specific primers targeting Bcl-2, MRP3, MRP7, P-glycoprotein, or the endogenous control U6 were purchased from Qiagen (Table 1). Quantitative reverse-transcription PCR (qRTPCR) was performed using miScript SYBR Green PCR Kit (Qiagen). All reactions were run in triplicate on a Bio-Rad C1000 thermal cycler (Bio-Rad). mRNA expression fold changes were calculated according to the $2^{-\Delta \Delta C T}$ method.

\section{Results}

\section{Cytotoxicity of ultrasound in PTX- resistant PC-3 cells}

Ultrasound-treated cells were incubated for 24 hours to determine the ultrasound treatment period that does not induce cytotoxicity in PTX-resistant PC-3 cells. The cell death rate was found to increase with prolonged ultrasound exposure, thereby demonstrating that ultrasound treatment killed PTX-resistant PC-3 cells. Therefore, 10 seconds of exposure time (less than $10 \%$ cytotoxicity) was used in subsequent experiments to avoid interference due to the cytotoxic effects induced by ultrasound treatment.

\section{Low-frequency ultrasound induces autophagy in PTX-resistant PC-3 cells}

Several autophagy assays were conducted to evaluate whether ultrasound induced autophagy in PTX-resistant PC-3 cells. First, autophagy was detected using a transmission electron microscope. As shown in Figure 1A, ultrasound-treated cells had swollen mitochondria, vacuoles, and presence of autophagosomes. Moreover, treatment with ultrasound in combination with PTX caused a significant increase in the number of autophagosomes in the PTX-resistant PC-3 cells. However, pretreatment of cells with Atg5 siRNA caused a decrease in the number of autophagosomes after treatment with ultrasound in combination with PTX.

Furthermore, we examined the effects of low-frequency ultrasound on the degree of autophagy by performing Western blot analysis. As shown in Figure 1B, the protein expression levels of Atg5 and LC3 II were significantly upregulated after 
A
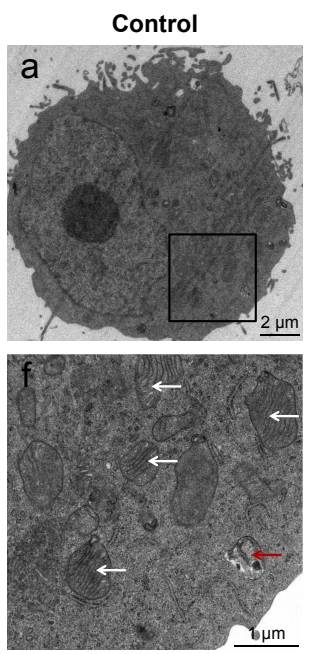

B

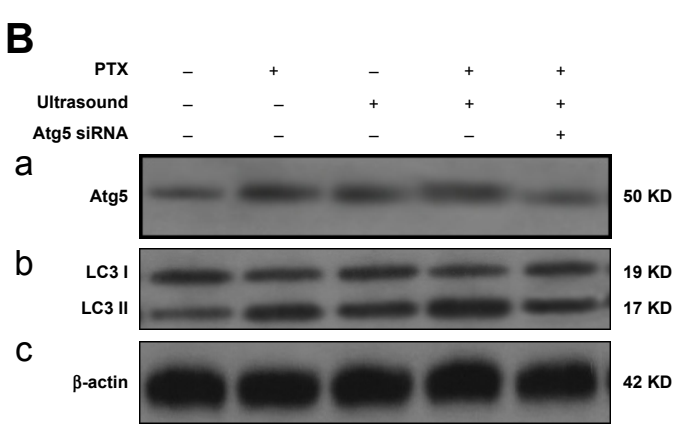

PTX
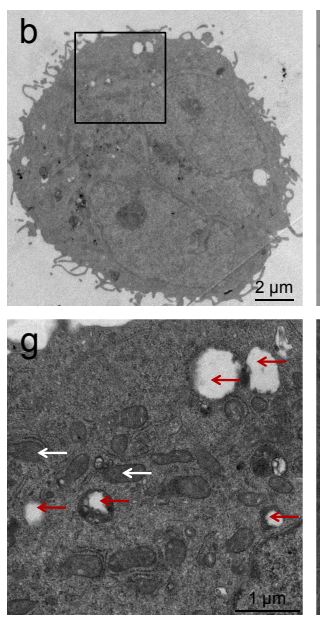

Ultrasound
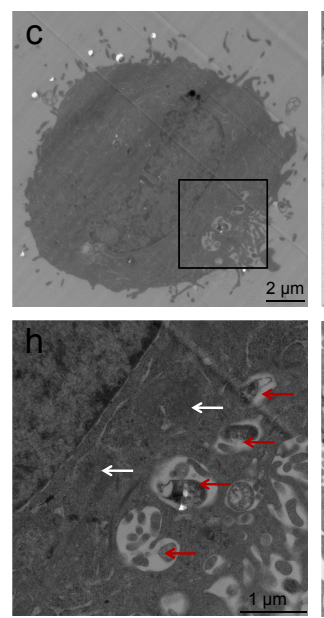

$\mathrm{d}_{0.8}$

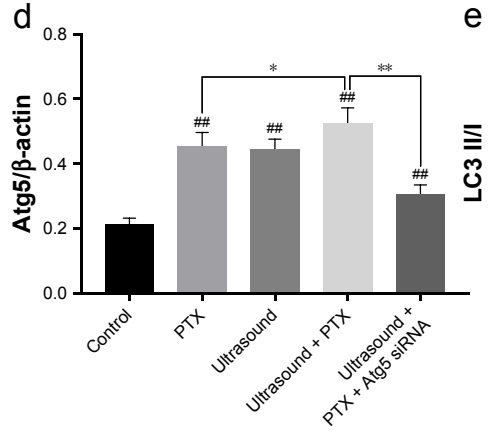

e
PTX + ultrasound
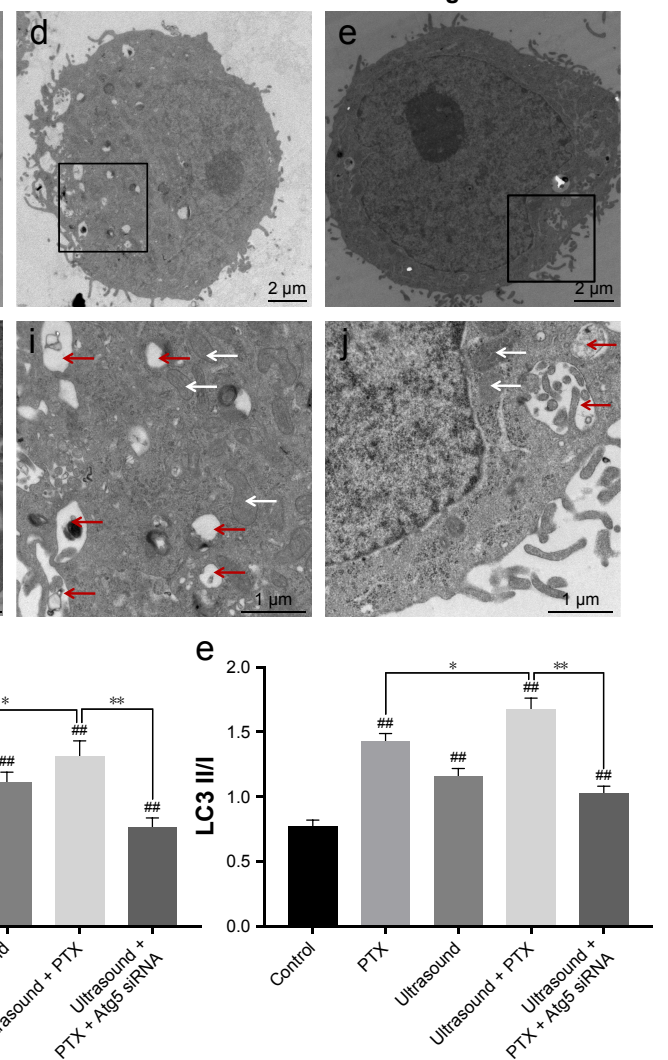

Figure I Effect of ultrasound treatment on autophagy in PTX-resistant PC-3 cells.

Notes: (A) Ultrastructural morphology and autophagy of paclitaxel-resistant PC-3 cells were evaluated using transmission electron microscopy at 24 hours after ultrasound treatment ( 10 seconds) with or without paclitaxel ( $91.44 \mathrm{nM}$ ) or Atg5 siRNA. The red and white arrows indicate autophagosomes and mitochondria, respectively. Figures (f), (g), (h), (i), and (j) are partial (black square) enlarged views of figures (a), (b), (c), (d), and (e) respectively. (B) Cells were untreated or treated with ultrasound (I0 seconds) in the presence or absence of Atg5 siRNA or paclitaxel (9l.44 nM) for 24 hours. Expression levels of Atg5 (a) and LC 3B (b) were assessed. Protein lysates were collected and assayed by Western blotting [loading control was $\beta$-actin(c)]. Figure (d) is the relative expression levels of Atg5 and figure (e) is the ratio of LC3II to LC3I. $* P<0.05$, $* * P<0.0$ I, $P<0.0$ I, compared with control group.

Abbreviation: PTX, paclitaxel.

treatment with ultrasound or cotreatment with ultrasound and PTX. Conversely, Atg5 and LC3 II protein levels were evidently downregulated after treatment with Atg5 siRNA to block autophagy in cells. The above-mentioned results showed that cotreatment with low-frequency ultrasound and PTX induced autophagy in PTX-resistant PC-3 cells, whereas treatment with Atg5 siRNA effectively inhibited autophagy.

\section{Low-frequency ultrasound induces apoptosis and enhances chemotherapy sensitivity in PTX-resistant PC-3 cells}

As shown in Figure 2A, flow cytometry was used to quantify the apoptotic rates of prostate cancer cell lines. Here, the apoptotic rates of PTX-resistant PC-3 cells treated with ultrasound combined with PTX were higher than those of PTX-resistant PC-3 cells treated with PTX alone. In addition, the apoptotic rates of PTX-resistant PC-3 cells transfected with Atg5 siRNA were significantly higher relative to those of cells treated with both ultrasound and PTX $(P<0.01)$.
Furthermore, CCK- 8 assay was performed to explore the effects of ultrasound on chemotherapy sensitivity in PTX-resistant PC-3 cells. We observed a significant dose-dependent increase in the PTX inhibition rates in prostate cancer cells. Moreover, the PTX inhibition rates of PTX-resistant PC-3 cells treated with ultrasound were higher than those of the control group and lower than those of PTX-resistant PC-3 cells treated with Atg5 siRNA and low-frequency ultrasound (Figure 2B).

Moreover, we analyzed the expression of genes that are known to be involved in the apoptosis and drug resistance. As shown in Figure 2C, the expression levels of Bcl-2, MPR3, MPR7, and P-glycoprotein were downregulated after treatment with ultrasound and PTX. Importantly, the expression levels of these genes were significantly downregulated in PTX-resistant PC-3 cells transfected with Atg5 siRNA compared with cells cotreated with ultrasound and PTX. The above-mentioned findings strongly suggested that low-frequency ultrasound increased PTX sensitivity 
A
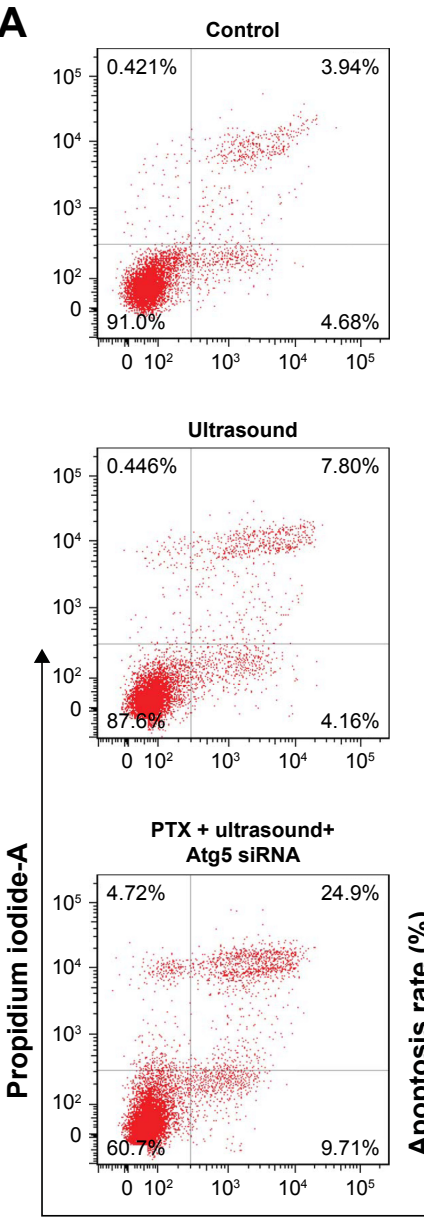

FITC-A
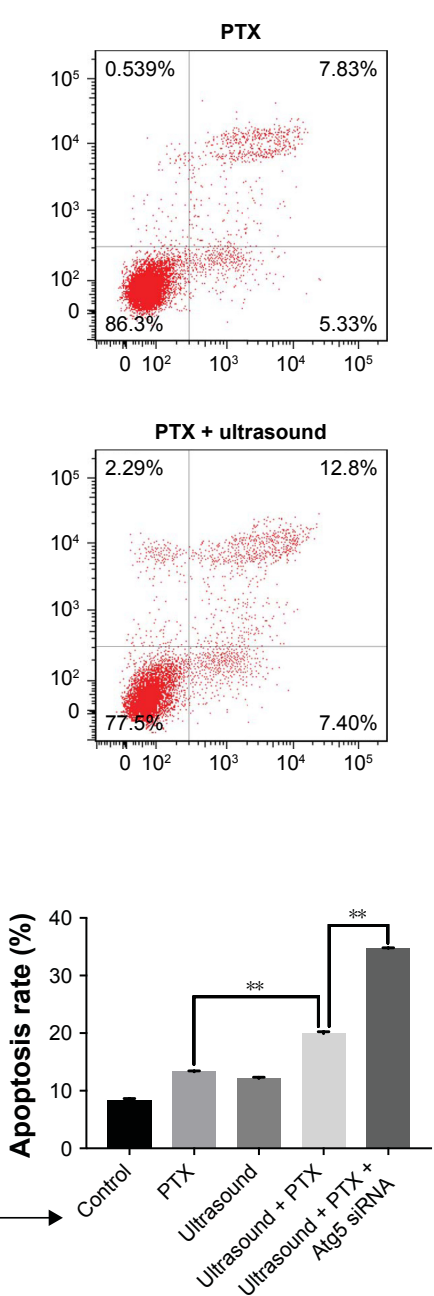

B

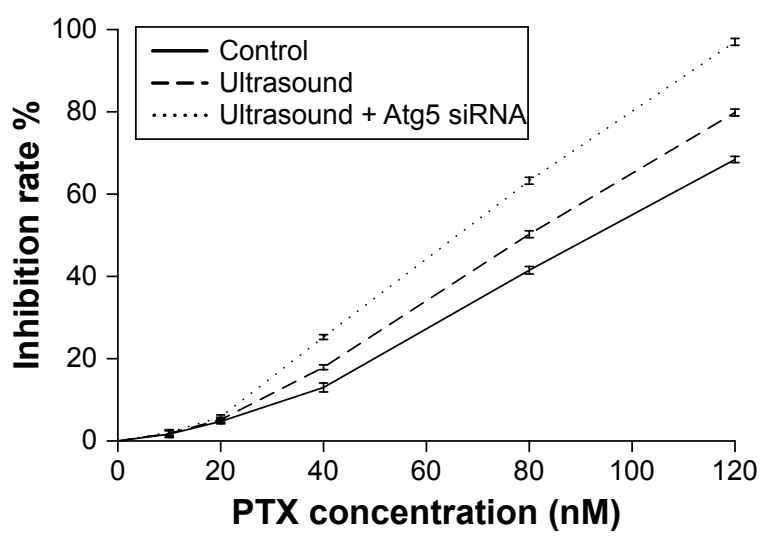

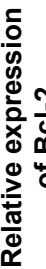
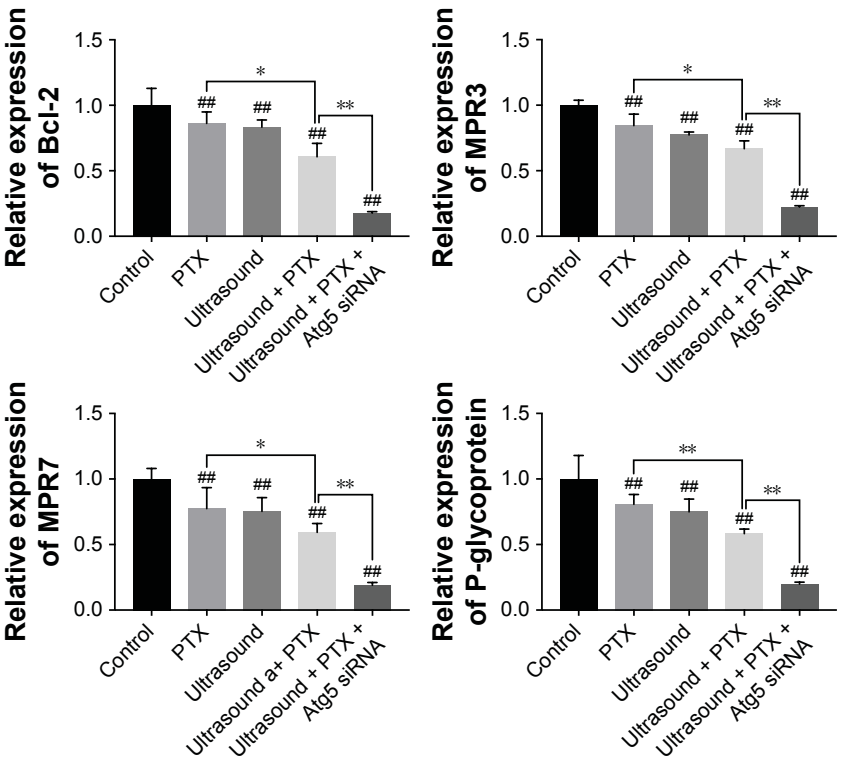

Figure 2 Effect of ultrasound treatment on apoptosis and chemotherapy sensitivity in PTX-resistant PC-3 cells.

Notes: PTX-resistant PC-3 cells were untreated or treated with ultrasound (10 seconds) in the presence or absence of Atg5 siRNA or PTX (9I.44 nM) for 24 hours, (A) stained with Annexin V-FITC and PI, then measured by flow cytometry. (B) PTX-resistant PC-3 cells treated with ultrasound (I0 seconds) in the presence or absence of Atg5 siRNA were treated with different concentrations of PTX for 24 hours. Cell inhibition was assessed via the CCK-8 assay. (C) Expression levels of Bcl-2, MPR3, MPR7, and P-glycoprotein were assessed by $\mathrm{qPCR}$. $* \mathrm{P}<0.05$, $* * \mathrm{P}<0.0 \mathrm{I}, \ldots \mathrm{P}<0.0 \mathrm{I}$, relative to the control group.

Abbreviations: FITC, fluorescein isothiocyanate; PI, propidium iodide; CCK-8, Cell-Counting Kit 8; qPCR, quantitative reverse-transcription PCR; PTX, paclitaxel.

by inducing apoptosis and downregulating the expression of resistance-related genes in PTX-resistant PC-3 cells. Ultrasound-induced autophagy was found to act as a cytoprotector during low-frequency ultrasound-mediated reversal of drug resistance.

\section{Low-frequency ultrasound induces apoptosis via the ER-mediated PI3K/AKT/ mTOR signaling pathway in PTX-resistant PC-3 cells}

To examine the effects of low-frequency ultrasound on endoplasmic reticulum stress (ERs), we performed Western blot analysis to evaluate the expression of GRP78, an ER marker protein. As shown in Figure 3, GRP78 protein expression levels were significantly upregulated by treatment with ultrasound or cotreatment with ultrasound and PTX. Furthermore, we elucidated the molecular mechanism underlying the interplay between ERs and apoptosis. As shown in Figure 3, protein expression levels of PI3K, p-AKT, mTORC1, Bcl-2, and p65 (NF-kB related protein) were significantly downregulated after treatment with ultrasound and further decreased after cotreatment with ultrasound and PTX. Interestingly, protein expression levels of GRP78 were significantly upregulated after treatment with Atg5 siRNA, whereas protein levels of PI3K, p-AKT, mTORC1, Bcl-2, and p65 were evidently decreased. Taken together, the observed changes in the expression levels of genes involved in the PI3K/AKT/mTOR signaling pathway indicated that ultrasound induces apoptosis via the ER-mediated PI3K/AKT/mTOR signaling pathway in PTX-resistant PC-3 cells. Therefore, inhibition of autophagy can potentially promote the $\mathrm{PI} 3 \mathrm{~K} / \mathrm{AKT} / \mathrm{mTOR}$ signaling pathway. 

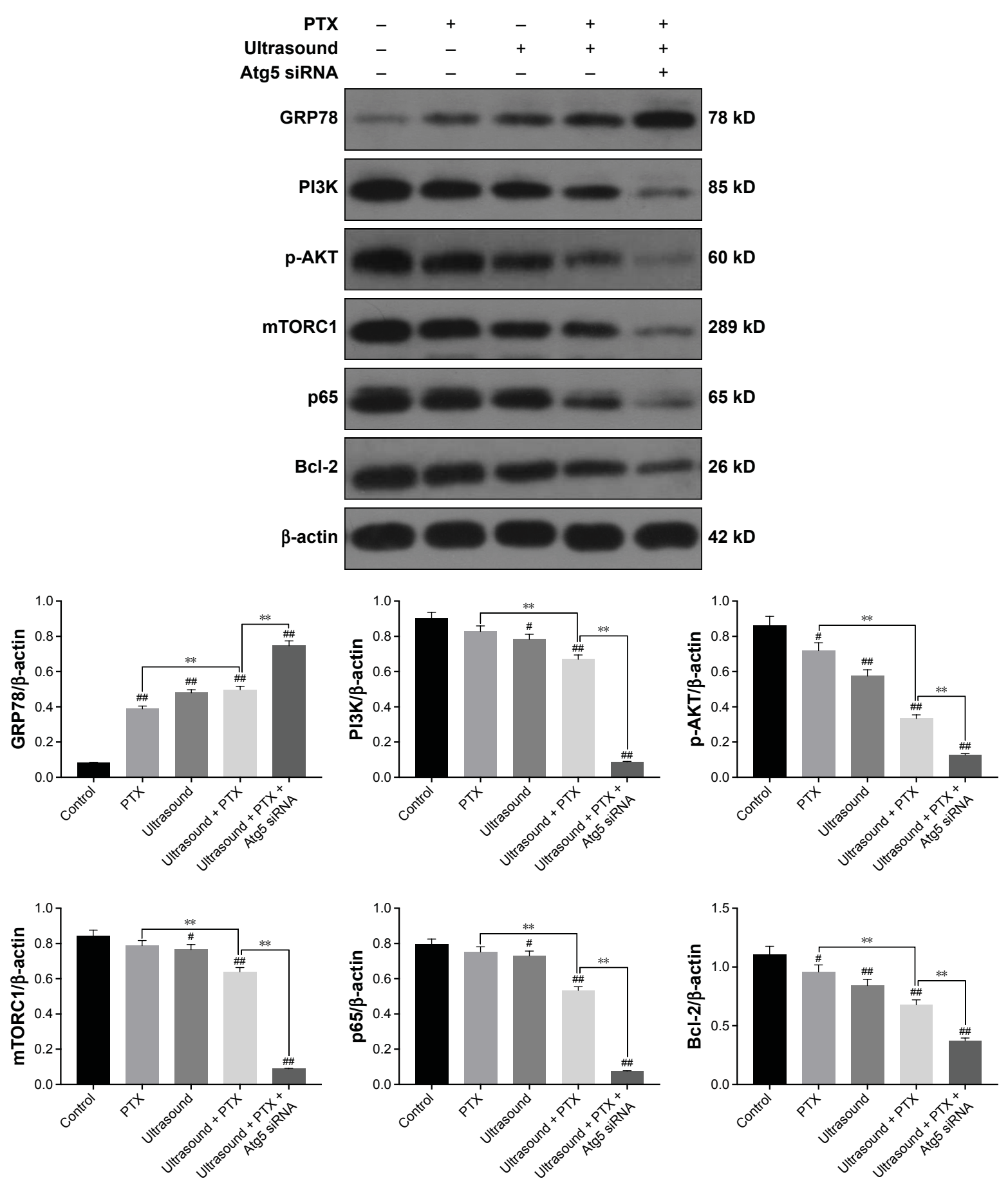

Figure 3 Effect of ultrasound treatment on ERs-mediated PI3K/Akt/mTOR signaling pathway in PTX-resistant PC-3 cells.

Notes: PTX-resistant PC-3 cells were untreated or treated with ultrasound (I0 seconds) in the presence or absence of Atg5 siRNA or PTX ( 91.44 nM) for 24 hours.

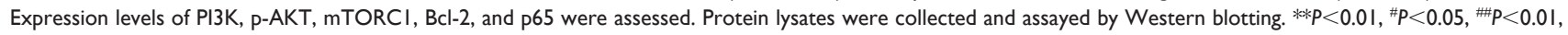
relative to the control group.

Abbreviations: PTX, paclitaxel; ERs, endoplasmic reticulum stress.

\section{Low-frequency ultrasound induces} autophagy via the ER-mediated PI3K/ AKT/mTOR signaling pathway in PTXresistant PC-3 cells

To examine the relationship between ERs and autophagy, PTX-resistant PC-3 cells were treated with 4-PBA, an ERs inhibitor. Autophagy was investigated using transmission electron microscopy. As shown in Figure 4A, the number of autophagosomes was significantly higher in ultrasoundtreated PTX-resistant PC-3 cells. However, the number of autophagosomes decreased after treatment with $10 \mathrm{mM}$ 4-PBA. Furthermore, we performed immunofluorescence 

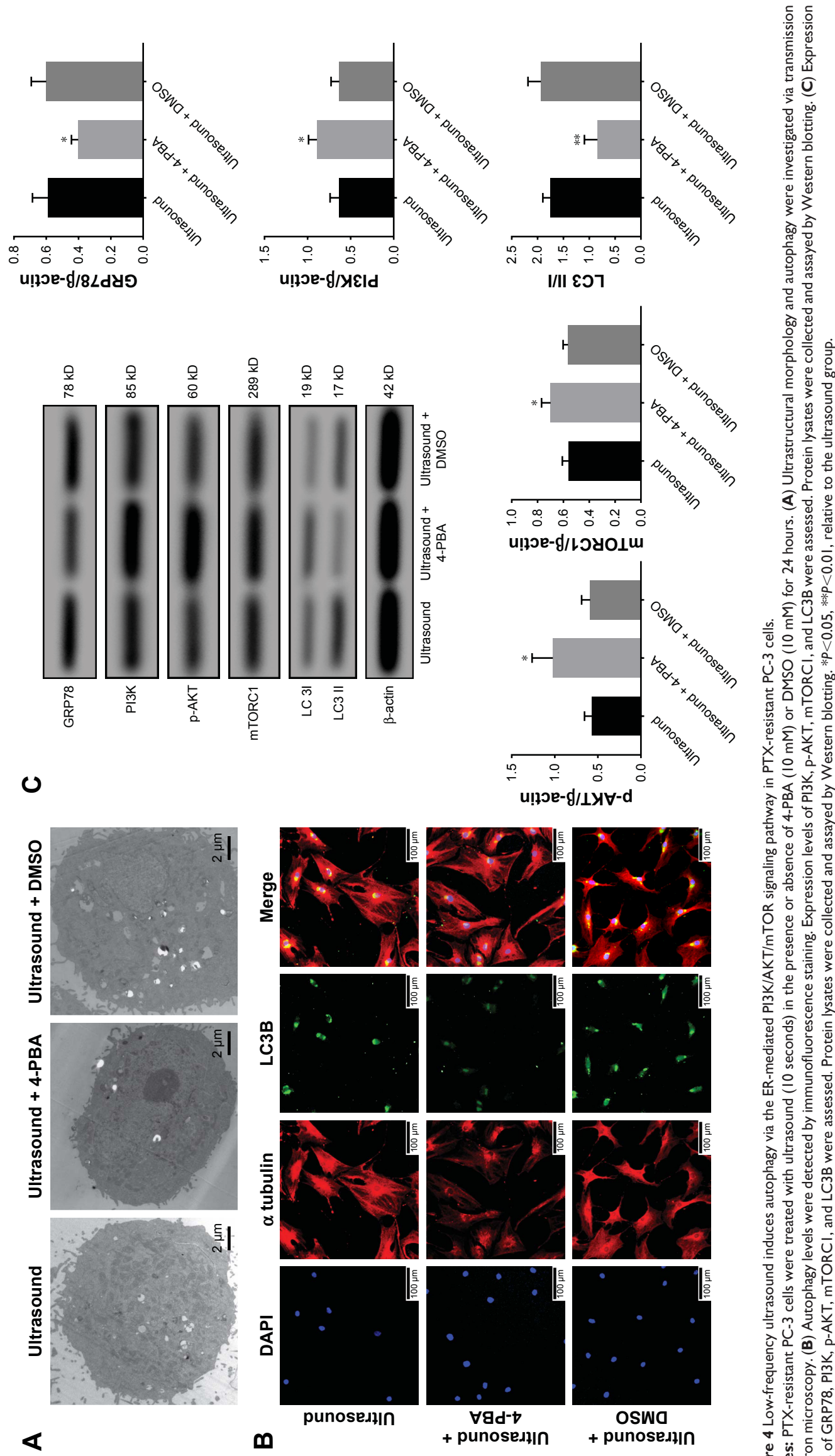

$\mathbf{m}$

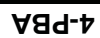

+ punosedi|
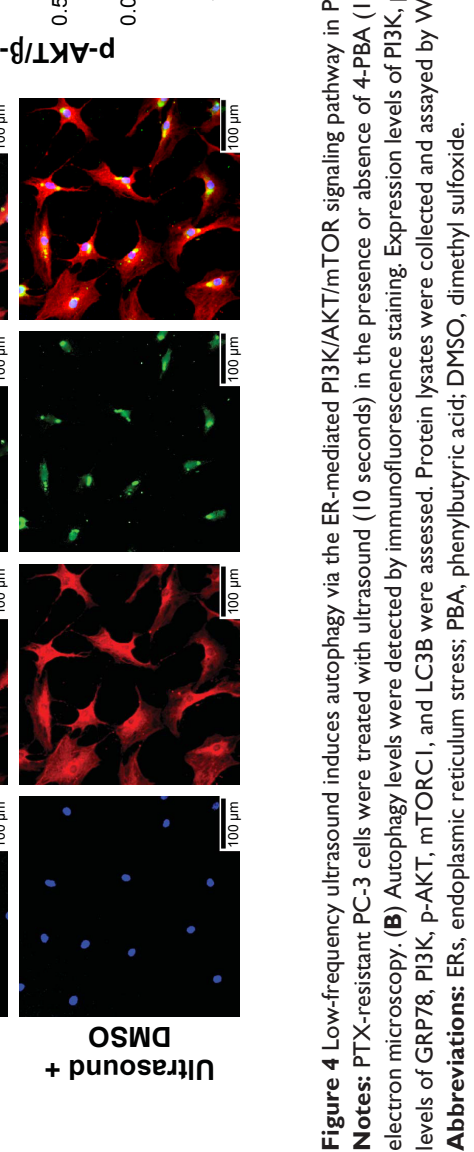
staining to monitor autophagy. As shown in Figure 4B, green fluorescence was emitted in ultrasound-treated cells. However, treatment of cells with 4-PBA reduced the intensity of green fluorescence signals after ultrasound treatment.

In addition, the interplay between ERs and autophagy was further investigated by evaluating the expression levels of proteins involved in the ER-mediated PI3K/AKT/ mTOR signaling pathway. As shown in Figure 4C, we observed a significant decrease in the expression of GRP78 and LC3II proteins, whereas PI3K, p-AKT, and mTORC1 expression levels increased after treatment with 4-PBA and ultrasound. Consistent with the above-mentioned results, ultrasound treatment was found to induce autophagy via the ER-mediated PI3K/AKT/mTOR signaling pathway in PTX-resistant PC-3 cells.

\section{Discussion}

Previous studies have demonstrated that low-frequency ultrasound can selectively increase the cell membrane permeabilities of tumor cells in breast cancer, ovarian cancer, and neuroblastoma. Simultaneously, low-frequency ultrasound can activate the mitochondria-caspase signaling pathway and downregulate the expression of ATP-binding cassette (ABC) transporter, to improve the sensitivity of tumors to chemotherapy drugs and reverse drug resistance (Wang, 2015 \#118). ${ }^{4}$ The present study examined the drug resistance-reversing effects of low-frequency ultrasound in PTX-resistant prostate cancer cells. Results revealed that autophagy in prostate cancer cells is induced by the administration of low-frequency ultrasound in combination with PTX. Treatment with Atg5 siRNA to inhibit autophagy was observed to downregulate the expression of the anti-apoptotic gene Bcl-2 and was accompanied by a significant increase in the apoptosis rate. MRP3, MRP7, and P-glycoprotein are members of the ABC transporter family, which pump chemotherapeutic drugs to reduce the sensitivity of PTX to cells. Inhibition of autophagy leads to the downregulation of transcription of these genes, accompanied by higher concentrations of intracellular chemotherapeutic drugs, which in turn significantly increases the efficiency of low-frequency ultrasound in reversing drug resistance. The above-mentioned findings suggested that autophagy can exert a protective effect against prostate cancer during low-frequency ultrasound-assisted PTX treatment.

The endoplasmic reticulum, a central organelle important for cellular function, is involved in secretion, as well as the folding, transfer, and post-translocational modification of the secretory proteins. Impaired function of the endoplasmic reticulum leads to the accumulation of misfolded and unfolded proteins in a process called ERs. ${ }^{9}$ Previous studies have shown that ERs can be induced by chemotherapeutic drugs. ${ }^{10}$ However, low-frequency ultrasound has rarely been reported to cause ERs. Our results indicated that ERs occurred in prostate cancer cells after low-frequency ultrasound irradiation during a non-toxic exposure period and can be associated with low-frequency ultrasound cavitation. Treatment of cancers with ultrasound causes oscillation of bubbles in the cells, which in turn leads to flow of the surrounding liquid and the mixture with the surrounding cytoplasm. Afterward, bubbles will continue to enlarge until implosion occurs. ${ }^{11}$ The function of the endoplasmic reticulum can be influenced by this process, resulting in ERs. Ranjan et al demonstrated that treatment of pancreatic cancer cells with pentafluoride can induce autophagy by triggering ERs. Furthermore, the autophagy levels were downregulated when ERs was blocked using ERs blockers or CHOP siRNA. ${ }^{12}$ Our experimental results revealed that treatment with low-frequency ultrasound irradiation induces ERs and increases autophagy levels. Treatment with low-frequency ultrasound combined with PTX aggravates ERs, thereby increasing autophagy levels. However, inhibition of ultrasonically induced autophagy was observed when cells were treated with the ERs inhibitor 4-phenylbutyrate. Interestingly, our current findings showed an evident increase in ERs when autophagy was inhibited. Autophagy can provide energy and materials in the process of "self-cannibalization" at the same time that ERs induces autophagy to recover the intracellular environment, thereby inhibiting ERs. In addition, other studies showed that autophagy can eliminate reactive oxygen species (ROS) and misfolded proteins to avoid their accumulation in cells. The accumulation of ROS and protein is considered to be an important factor in inducing ERs. ${ }^{13}$

Yang et al treated ovarian cancer cells with tunicamycin, and their results revealed that ERs could inhibit PI3K/AKT/ mTOR signaling to induce apoptosis in human ovarian cancer cells. ${ }^{14}$ The PI3K/AKT/mTOR is a classical signaling pathway that acts as a critical regulator of autophagy. The activation of class I PI3 kinase (PI3K) can phosphorylate ptdIns(4,5)P2 and form PtdIns(3,4,5)P3 in the plasma membrane. Meanwhile, class I PI3 kinase (PI3K) can recruit protein kinase $\mathrm{B}$ and phosphoinositide-dependent protein kinase 1; co-recruitment of these two kinases leads to the activation of AKT. Subsequently, phosphorylation of TSC1 leads to the accumulation of RHEB GTP, which can be promoted by activated AKT, ${ }^{15-17}$ whereas the former can activate the mTOR. ${ }^{18,19}$ Furthermore, mTOR can activate the downstream ribosomal protein S6 (p7s6), and S6 (p7s6) 
phosphorylation can inhibit autophagy. ${ }^{20,21}$ Our experimental results demonstrated that the PI3K/AKT/mTOR pathway is inhibited when low-frequency ultrasound treatment was applied to induce ERs. Meanwhile, the combined use of lowfrequency ultrasonic and PTX leads to the downregulation of PI3K, p-Akt, and mTORC1, which is accompanied by increased ERs. ERs was further enhanced by the application of ATG5 siRNA to inhibit autophagy, leading to marked inhibition of the PI3K/AKT/mTOR pathway. Inhibition of ERs corresponds to a weaker inhibitory effect of ultrasound treatment on the PI3K/AKT/mTOR pathway. Therefore, low-frequency ultrasound is assumed to induce autophagy by triggering ERs, which acts by inhibiting the PI3K/AKT/ mTOR signaling pathway. Cotreatment with low-frequency ultrasound and PTX enhances ERs, inhibits the PI3K/AKT/ mTOR pathway, and increases autophagy levels. By contrast, upon inhibition of autophagy, the regulatory effect on the intracellular environment is weakened along with furthur increse in ERs, thus leading to stronger inhibitory effect on the PI3K/AKT/mTOR pathway.

mTOR is the primary regulator of cell growth that participates in drug resistance in tumor cells and can inhibit tumor apoptosis by regulating the expression of NF- $\kappa \mathrm{B}$ and $\mathrm{ABC}$ transporters. ${ }^{22}$ Our experimental results demonstrated that downregulation of $\mathrm{mTORC} 1$ expression is associated with the downregulation of P65, MRP3, MRP7, and P-glycoprotein levels. On the other hand, the PI3K/Akt signal transduction pathway is considered to be the primary pathway that mediates the survival of tumor cells. AKT can phosphorylate several of its downstream substrates to facilitate the growth and proliferation of tumor cells, inhibit cell apoptosis, and enhance tumor cell resistance against chemotherapy and radiotherapy in a process mediated by the Bcl-2 family and the apoptosis inhibitory protein family. In addition, our findings demonstrated that the transcriptional activity of NF- $\kappa B$ can be increased via phosphorylation of eNOS. ${ }^{23,24}$

\section{Conclusion}

In summary, treatment with low-frequency ultrasound can trigger ERs, which in turn inhibits the PI3K/AKT/mTOR signal transduction pathway to downregulate the transcriptional activity of $\mathrm{ABC}$ transporter Bcl-2 and NF- $\mathrm{\kappa B}$. In turn, the intracellular concentrations of PTX are increased, thereby promoting apoptosis and reversing drug resistance. On the other hand, downregulation of the PI3K/AKT/mTOR signal transduction pathway by ERs can induce autophagy of PTXresistant cells in prostate cancer in the process of reversing the resistance of tumor cells by low-frequency ultrasound.

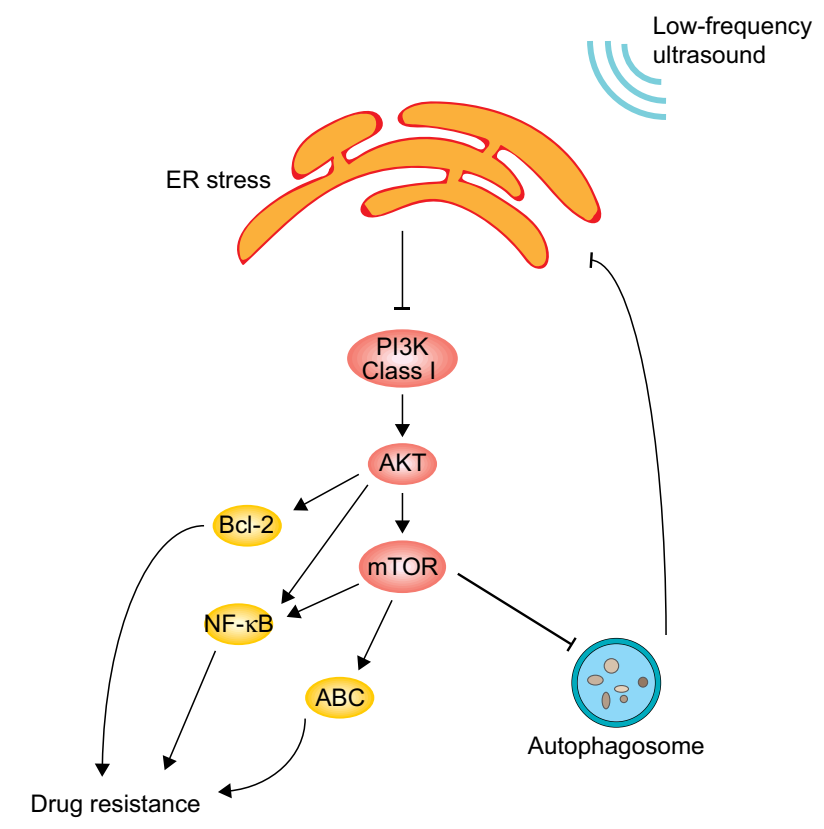

Figure 5 Schematic showing the molecular mechanisms underlying the effects of low-frequency ultrasound in PTX-resistant PC-3 cells.

Notes: Ultrasound induces apoptosis and enhances chemotherapy sensitivity through ER-mediated PI3K/AKT/mTOR signaling pathway in PTX-resistant PC-3 cells. Ultrasound-induced autophagy acts as a cytoprotector during low-frequency ultrasound-mediated reversal of drug resistance. Ultrasound induces autophagy by ER-mediated PI3K/AKT/mTOR signaling pathway in PTX-resistant PC-3 cells. However, inhibition of autophagy can promote PI3K/AKT/mTOR signaling.

Abbreviation: ER, endoplasmic reticulum.

On the other hand, autophagy can, in turn, inhibit ERs by eliminating ROS and misfolded proteins. Autophagy inhibition promotes ERs, thereby further inhibiting the PI3K/AKT/ mTOR signal transduction pathway. Consequently, a further decrease in the transcriptional activity of $\mathrm{ABC}$ transporter $\mathrm{Bcl}-2$ and NF- $\mathrm{\kappa B}$ is accompanied by an increase in the intracellular concentrations of PTX and promotion of cell apoptosis. Hence, reversal of drug resistance is significantly improved by low-frequency ultrasound (Figure 5).

\section{Acknowledgment}

The study was sponsored by the National Natural Science Foundation of China (Grant 81172443) and the Shenzhen Science and Technology Innovation Committee (Grant 20180077) and Shenzhen University General Hospital Science and Technology Talent Promotion Program (Grant SUGH-2018-001).

\section{Disclosure}

The authors report no conflicts of interest in this work.

\section{References}

1. Siegel RL, Miller KD, Jemal A, Statistics C. Cancer statistics, 2018. CA Cancer J Clin. 20182018;68(1):7-30. 
2. Cornford P, Bellmunt J, Bolla M, et al. EAU-ESTRO-SIOG guidelines on prostate cancer. Part II: treatment of relapsing, metastatic, and castration-resistant prostate cancer. Eur Urol. 2017;71(4):630-642.

3. Costley D, Mc Ewan C, Fowley C, et al. Treating cancer with sonodynamic therapy: a review. Int J Hyperthermia. 2015;31(2):107-117.

4. Wang X, Jia Y, Su X, et al. Combination of protoporphyrin IX-mediated sonodynamic treatment with doxorubicin synergistically induced apoptotic cell death of a multidrug-resistant leukemia K562/DOX cell line. Ultrasound Med Biol. 2015;41(10):2731-2739.

5. Wang P, Leung AW, Xu C. Low-intensity ultrasound-induced cellular destruction and autophagy of nasopharyngeal carcinoma cells. Exp Ther Med. 2011;2(5):849-852.

6. Wang YU, Chen YN, Zhang W, Yang YU, Shen E, Hu B. Upregulation of Beclin-1 expression in DU-145 cells following low-frequency ultrasound irradiation combined with microbubbles. Oncol Lett. 2015; 10(4):2487-2490.

7. Amaravadi R, Kimmelman AC, White E. Recent insights into the function of autophagy in cancer. Genes Dev. 2016;30(17):1913-1930.

8. Zhang W, Wan X, Liu Z, Xiao L, Huang H, Liu X. The emerging role of oxidative stress in regulating autophagy: applications of cancer therapy. Cell Mol Biol. 2017;63(4):67-76.

9. Mohamed E, Cao Y, Rodriguez PC. Endoplasmic reticulum stress regulates tumor growth and anti-tumor immunity: a promising opportunity for cancer immunotherapy. Cancer Immunol Immunother. 2017;66(8): 1069-1078

10. Iurlaro R, Muñoz-Pinedo C. Cell death induced by endoplasmic reticulum stress. Febs J. 2016;283(14):2640-2652.

11. Frulio N, Trillaud H, Deckers R, Lepreux S, Moonen C, Quesson B. Influence of ultrasound induced cavitation on magnetic resonance imaging contrast in the rat liver in the presence of macromolecular contrast agent. Invest Radiol. 2010;45(5):282-287.

12. Ranjan A, German N, Mikelis C, Srivenugopal K, Srivastava SK. Penfluridol induces endoplasmic reticulum stress leading to autophagy in pancreatic cancer. Tumour Biol. 2017;39(6):1010428317705517.

13. Gump JM, Thorburn A. Autophagy and apoptosis: what is the connection? Trends Cell Biol. 2011;21(7):387-392.
14. Yang N, Qu YJ, Cheng Y, et al. Endoplasmic reticulum stress regulates proliferation, migration and invasion of human ovarian cancer SKOV3 cells through PI3K/AKT/mTOR signaling pathway. Cancer Biomark. 2017;19(3):263-269.

15. Mabuchi S, Kuroda H, Takahashi R, Sasano T. The PI3K/AKT/mTOR pathway as a therapeutic target in ovarian cancer. Gynecol Oncol. 2015; 137(1):173-179.

16. Danielsen SA, Eide PW, Nesbakken A, Guren T, Leithe E, Lothe RA. Portrait of the PI3K/AKT pathway in colorectal cancer. Biochim Biophys Acta. 2015;1855(1):104-121.

17. Bertacchini J, Heidari N, Mediani L, et al. Targeting PI3K/AKT/mTOR network for treatment of leukemia. Cell Mol Life Sci. 2015;72(12): 2337-2347.

18. Filippi-Chiela EC, Viegas MS, Thomé MP, Buffon A, Wink MR, Lenz G. Modulation of autophagy by calcium signalosome in human disease. Mol Pharmacol. 2016;90(3):371-384.

19. Li Y, Zhang J, Chen X, et al. Molecular machinery of autophagy and its implication in cancer. Am J Med Sci. 2012;343(2):155-161.

20. Bhat P, Kriel J, Shubha Priya B, Basappa, Shivananju NS, Loos B. Modulating autophagy in cancer therapy: advancements and challenges for cancer cell death sensitization. Biochem Pharmacol. 2018;147: $170-182$.

21. Ruf S, Heberle AM, Langelaar-Makkinje M, et al. PLK1 (polo like kinase 1) inhibits MTOR complex 1 and promotes autophagy. Autophagy. 2017;13(3):486-505.

22. Chandrika G, Natesh K, Ranade D, Chugh A, Shastry P. Suppression of the invasive potential of glioblastoma cells by mTOR inhibitors involves modulation of NFKB and PKC- $\alpha$ signaling. Sci Rep. 2016;6:22455.

23. Zou Y, Li Q, Jiang L, et al. DNA hypermethylation of CREB3L1 and Bcl-2 associated with the mitochondrial-mediated apoptosis via PI3K/ Akt pathway in human BEAS-2B cells exposure to silica nanoparticles. PLoS One. 2016;11(6):e0158475.

24. Bao G, Li C, Qi L, Wang N, He B. Tetrandrine protects against oxygenglucose-serum deprivation/reoxygenation-induced injury via PI3K/ $\mathrm{AKT} / \mathrm{NF}-\kappa \mathrm{B}$ signaling pathway in rat spinal cord astrocytes. Biomed Pharmacother. 2016;84:925-930.
OncoTargets and Therapy

\section{Publish your work in this journal}

OncoTargets and Therapy is an international, peer-reviewed, open access journal focusing on the pathological basis of all cancers, potential targets for therapy and treatment protocols employed to improve the management of cancer patients. The journal also focuses on the impact of management programs and new therapeutic agents and protocols on

\section{Dovepress}

patient perspectives such as quality of life, adherence and satisfaction The manuscript management system is completely online and includes a very quick and fair peer-review system, which is all easy to use. Visit http://www.dovepress.com/testimonials.php to read real quotes from published authors. 